\title{
Enhancing cellular uptake of activable cell-penetrating peptide-doxorubicin conjugate by enzymatic cleavage
}

This article was published in the following Dove Press journal:

International Journal of Nanomedicine

24 March 2012

Number of times this article has been viewed

\author{
Nian-Qiu Shi \\ Wei Gao \\ Bai Xiang \\ Xian-Rong Qi
}

Department of Pharmaceutics, School of Pharmaceutical Sciences, Peking University, Beijing, People's Republic of China
Correspondence: Xian-Rong Qi Department of Pharmaceutics, School of Pharmaceutical Sciences, Peking University, 38 Xueyuan Road, Beijing I00I9I, People's

Republic of China

Tel/fax +86 I0 8280I584

Email qixr200I@yahoo.com.cn
Abstract: The use of activable cell-penetrating peptides (ACPPs) as molecular imaging probes is a promising new approach for the visualization of enzymes. The cell-penetrating function of a polycationic cell-penetrating peptide (CPP) is efficiently blocked by intramolecular electrostatic interactions with a polyanionic peptide. Proteolysis of a proteinase-sensitive substrate present between the CPP and polyanionic peptide affords dissociation of both domains and enables the activated CPP to enter cells. This ACPP strategy could also be used to modify antitumor agents for tumor-targeting therapy. Here, we aimed to develop a conjugate of ACPP with antitumor drug doxorubicin (DOX) sensitive to matrix metalloproteinase-2 and -9 (MMP-2/9) for tumor-targeting therapy purposes. The ACPP-DOX conjugate was successfully synthesized. Enzymatic cleavage of ACPP-DOX conjugate by matrix metalloproteinase (MMP)-2/9 indicated that the activation of ACPP-DOX occurred in an enzyme concentration-dependent manner. Flow cytometry and laser confocal microscope studies revealed that the cellular uptake of ACPP-DOX was enhanced after enzymatic-triggered activation and was higher in HT-1080 cells (overexpressed MMPs) than in MCF-7 cells (under-expressed MMPs). The antiproliferative assay showed that ACPP had little toxicity and that ACPP-DOX effectively inhibited HT-1080 cell proliferation. These experiments revealed that the ACPP-DOX conjugate could be triggered by MMP-2/9, which enabled the activated CPP-DOX to enter cells. ACPP-DOX conjugate may be a potential prodrug delivery system used to carry antitumor drugs for MMP-related tumor therapy.

Keywords: activable cell-penetrating peptide, matrix metalloproteinase, proteinase-sensitive substrate, cellular uptake, antiproliferative, enzymatic cleavage, tumor extracellular environment

\section{Introduction}

The use of cell-penetrating peptides (CPPs) has become one of the most popular and efficient techniques for achieving intracellular access. Commonly used CPPs include TAT, polyarginine, penetratin, and transportan. These CPPs have successfully delivered proteins, ${ }^{1}$ nucleic acids, ${ }^{2}$ small molecule therapeutics, ${ }^{3}$ quantum dots, ${ }^{4}$ and magnetic resonance imaging contrast agents ${ }^{5}$ into cells. With the increased interest in CPP-mediated cargo delivery, CPPs have been applied in antitumor agent delivery. Doxorubicin (DOX) is a potent anthracycline used for the treatment of various types of cancer. It causes cytotoxicity through topoisomerase II-mediated DNA breaks. Some publications ${ }^{6,7}$ have reported that CPP-DOX conjugates displayed excellent therapeutic efficacy for related tumor therapy. Efficient induction of apoptosis by CPP-DOX was found in the human breast cancer cell line MDA-MB-231. ${ }^{8,9}$ In addition, various CPPdrug conjugates have also been developed to improve antitumor efficacy. ${ }^{10,11}$ 
However, the majority of known CPPs are not cell or tissue specific; CPPs and their attached therapeutic molecules are dispersed throughout almost all of the body irrespective of the method of administration. This property remains the major drawback for CPP application as drug delivery systems. ${ }^{12-14}$

The enzyme-sensitive substrate strategy is explorative and utilizes the stimulation of the unique tumor extracellular environment based on the principle of proteolysis. Proteolysis is a simple hydrolytic process that separates two adjacent amino acid residues at the amide bond and is triggered by proteases. ${ }^{15}$ Enzyme cleavable conjugates, containing enzyme-sensitive substrate, mainly take advantage of the selectivity and specificity of enzymes that are differentially active in the immediate environment of tumor cells.

Activable cell-penetrating peptides (ACPPs) containing polycationic CPPs, enzyme-sensitive substrate, and polyanionic inhibitory domains have been developed by Tsien's group as molecular imaging probes in tumor detection and intraoperative guidance. ${ }^{16-20}$ The ACPPs are inactive in the circulation since the cell-penetrating function of CPP is efficiently blocked by intramolecular electrostatic interactions with a polyanionic peptide. Proteolysis of a cleavable enzyme-sensitive substrate present between the CPP and polyanionic peptide by specific enzymes overexpressed on the disease site affords dissociation of both domains and enables the activated CPP to enter cells. This ACPP approach offers ways to address the major issue that CPPs lack cell specificity by means of the selective trigger of proteases in diseased tissue, and can be used for tumor-targeting therapy. It is interesting and meaningful to explore whether ACPPdrug conjugates can be exploited for targeted drug delivery via enhanced targeting ability.

Here, we aimed to develop an ACPP-DOX conjugate sensitive to matrix metalloproteinases (MMPs) for tumor-targeting therapy purposes. MMPs are one type of frequently used cleavable enzyme that are associated with tumor disease. The ACPP-DOX conjugate delivery system involves the polycationic domain (CPP), the cleavable MMP-2/9-sensitive substrate, the polyanionic domain (inhibitory or attenuating sequence), and DOX. The proposed mechanism of intracellular access enhancement is exhibited in Figure 1A. This ACPP-DOX delivery system was temporarily inactive in circulation and in non- or under-expressed MMP tissue and subsequently activated by overexpressed MMP-2/9 in targets where cargo could be released. The design, synthesis, and validation of the selectively triggered characterizations of this ACPP-DOX conjugate by extra MMPs and endogenous MMPs were determined by high-performance liquid chromatography (HPLC), flow cytometry, and confocal microscopy. To examine the effect of ACPP-DOX, DOX, and ACPP on cell growth and viability, the antiproliferative assay was measured on HT-1080 and MCF-7 cells. The novel ACPP-DOX delivery system provides a new choice for MMP-related tumor therapy.

\section{Materials and methods \\ Materials}

DOX (>99\%) was kindly donated by Hisun Pharmaceutical Co, Ltd, (Zhejiang, People's Republic of China). $\mathrm{N}$-succinimidyl 3-maleimidopropionate (SMP; >98\%) was purchased from Jiaxing Biomatrix Co, Ltd, (Zhejiang, People's Republic of China). Trypsin was obtained from Amresco Inc (Solon, OH). 3-(4,5-dimethylthiazol-2-yl)-2,5diphenyltetrazolium bromide (MTT) and collagenase IV were purchased from Sigma-Aldrich (St Louis, MO). Penicillin, streptomycin, and Hoechst 33258 were provided by Macgene Co, Ltd, (Beijing, People's Republic of China), while 4-aminophenylmercuric acetate (APMA) was purchased from Merck Co, Ltd, (Darmstadt, Germany). MMP-2 was procured from PeproTech EC Ltd (London, UK). All other chemicals were of analytic or HPLC grade.

The ACPP $\left(\mathrm{H}_{2} \mathrm{~N}\right.$-DGGDGGDGGDGPLGLAGrrrrrrrrC$\mathrm{COOH}$ [the uppercase letters represent L-amino acids, while the lowercase letters represent D-amino acids]) was custom synthesized via a standard Fmoc solid-phase peptide synthesis method by Shanghai GL Biochem Co, Ltd, (Shanghai, People's Republic of China). The purity of ACPP was 97.9\%. The correct product was confirmed by electrospray ionization mass spectroscopy (ESI-MS): $m / z, 2895.2$ (calculated), 2895.57 (observed, $[\mathrm{M}+3 \mathrm{H}]^{3+}:$ 966.19), 2896.18 (observed, $[\mathrm{M}+2 \mathrm{H}]^{2+}:$ 1449.09).

\section{Cell lines}

HT-1080 (human fibrosarcoma) cells were purchased from the Cell Culture Centre, Peking Union Medical College (Beijing, People's Republic of China). The cells were cultured in Minimum Essential Medium with Earle's salts, L-glutamine, nonessential amino acid (Macgene, Beijing, People's Republic of China), 10\% fetal bovine serum (FBS), 100 units $/ \mathrm{mL}$ penicillin, and $100 \mu \mathrm{g} / \mathrm{mL}$ streptomycin.

MCF-7 (human breastadenocarcinoma) cells were obtained from the Institute of Hematology and Blood Diseases Hospital (Tianjin, China). The cells were maintained in Dulbecco's Modified Eagle's Medium with glucose, sodium pyruvate, 

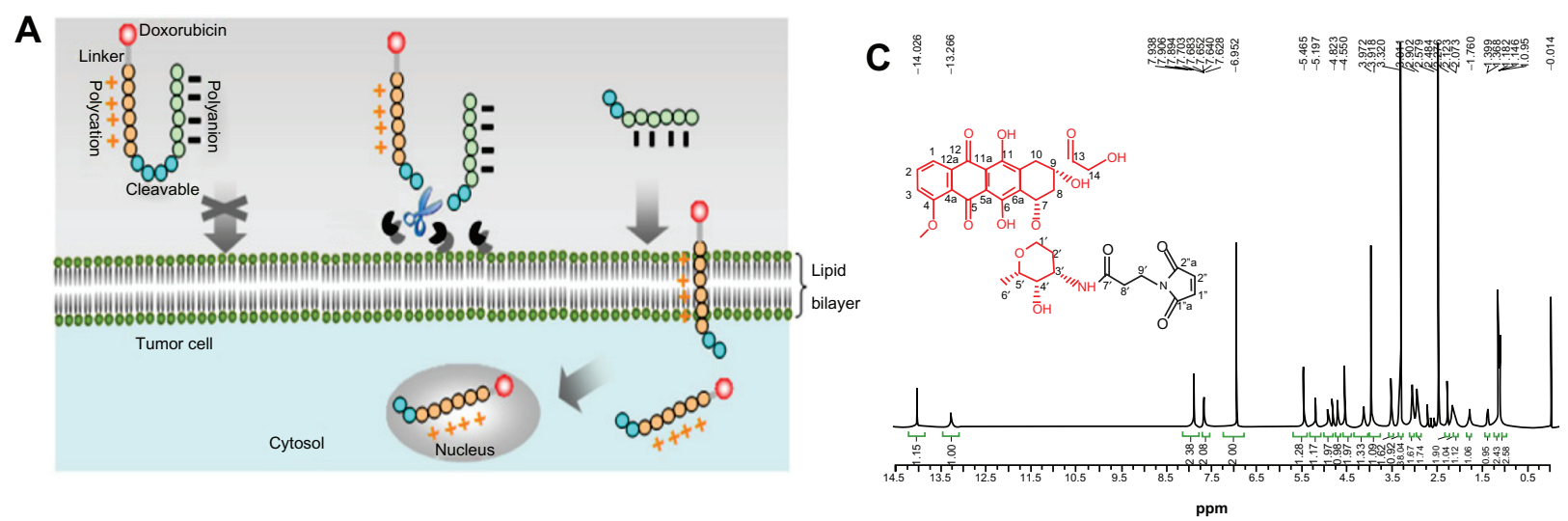
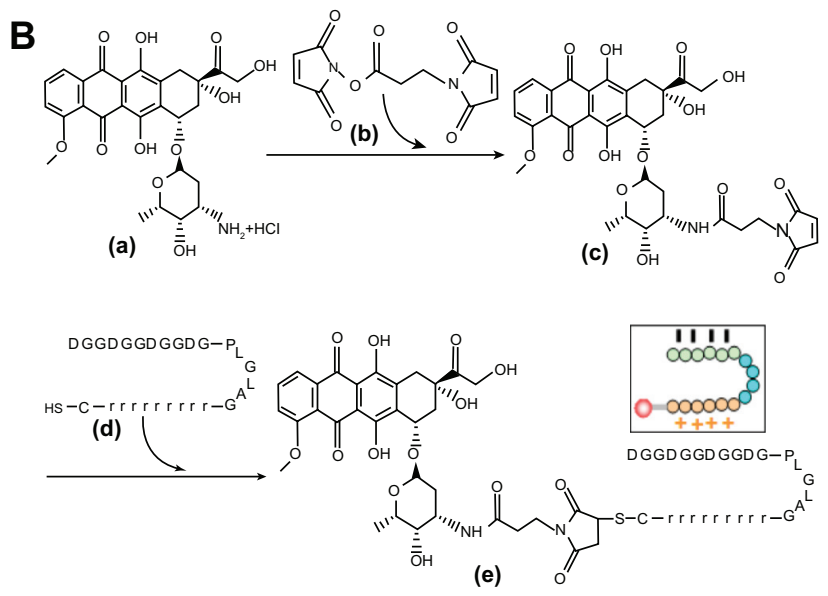

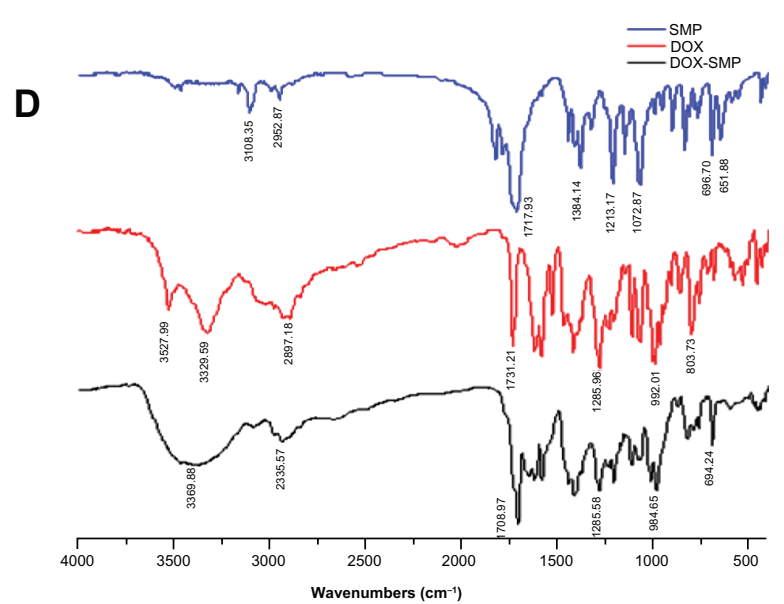

Figure I (A) Proposed schematic diagram of ACPP-DOX conjugate for antitumor drug delivery. ACPP-DOX conjugate is inactivated initially and circulates freely in vivo. When the ACPP-DOX conjugate accesses the tumor tissue, it is triggered and activated by overexpressed extracellular MMPs (MMP-2/9). The CPP-DOX releases and translocates into cells. The ACPP-DOX conjugate includes a cargo of doxorubicin, polycational CPP, polyanional attenuating peptides, and MMP-specific substrates. (B) Synthesis scheme of ACPP-DOX conjugates: (a) doxorubicin (DOX), (b) N-succinimidyl-3-maleimidopropionate (SMP), (c) DOX-SMP, (d) ACPP, an activatable cellpenetrating peptide. (e) ACPP-DOX, a derivative of DOX linked with the ACPP. (C) 'H-NMR spectrum of DOX-SMP. (D) FT-IR spectra of SMP, DOX, and DOX-SMP. Abbreviations: ACPP-DOX, activable cell-penetrating peptide-doxorubicin; CPP, cell-penetrating peptide; MMP, matrix metalloproteinase.

L-glutamine, and 4-(2-hydroxyethyl)-1-piperazineethanesulfonic acid (Macgene, Beijing, People's Republic of China) supplemented with $10 \% \mathrm{FBS}, 100$ units/mL penicillin, and $100 \mu \mathrm{g} / \mathrm{mL}$ streptomycin.

All the cells were cultured in an incubator maintained at $37^{\circ} \mathrm{C}$ with $5 \% \mathrm{CO}_{2}$ under fully humidified conditions. All experiments were performed on cells in the exponential growth phase.

\section{Synthesis of ACPP-DOX conjugate}

ACPP-DOX conjugate was synthesized by coupling ACPP with DOX using a linker of SMP. Thus, DOX-SMP was prepared first. The general synthetic scheme is depicted in Figure 1B. Briefly, DOX (43.5 mg, 1 Eq), SMP (22.0 mg, 1.1 Eq), and triethylamine (TEA; $2 \mathrm{Eq}$ ) were dissolved in $8 \mathrm{~mL}$ of dimethylformamide (DMF) and stirred at room temperature for 2 hours, while the progress of the reaction was monitored by thin-layer chromatography (TLC; chloroform:methanol: ammonia $=70: 30: 3$ ). The reaction solution was treated with $50 \mathrm{~mL}$ of cold anhydrous diethyl ether and the resulting precipitate was washed twice with $5 \mathrm{~mL}$ of anhydrous diethyl ether. The red solid that was obtained was separated from the solvent by centrifugation. The product was dried to give a $64.3 \%$ yield in red crystals.

Secondly, the ACPP-DOX was synthesized. DOX-SMP (2.6 mg, $1 \mathrm{Eq})$, ACPP (10 mg, $1 \mathrm{Eq})$, TEA (10 $\mu \mathrm{L}, 21 \mathrm{Eq})$, and $1 \mathrm{~mL}$ of DMF were mixed and stirred at room temperature for 2 hours, while the progress of the reaction was monitored using TLC and HPLC. The reaction solution was treated with $10 \mathrm{~mL}$ of cold anhydrous diethyl ether and the resulting precipitate was washed twice with $5 \mathrm{~mL}$ of anhydrous diethyl ether. The resulting red solid was separated from the solvent by centrifugation, and then the product was dried to give a $78.8 \%$ yield in red crystals. 


\section{Enzymatic-triggered cleavage of ACPP-DOX by MMPs}

To analyze the enzymatic cleavage of the matrix metalloproteinase (MMP)-sensitive conjugates, ACPP-DOX cleavage was studied in the presence of MMP-2/9. Collagenase IV (containing MMP-2/9) was activated with the 2.5 mMAPMA solution for 1 hour at $37^{\circ} \mathrm{C}$. ACPP-DOX stock solution was mixed with activated collagenase IV and incubated at $37^{\circ} \mathrm{C}$. The aliquots were removed between 1 hour and 4 hours and analyzed by HPLC. The HPLC system was equipped with a Waters 2487 Dual Wavelength Absorbance Detector and 1525 Pump (Milford, MA), and the wavelength was set at $220 \mathrm{~nm}$. To determine ACPP-DOX conjugate and hydrolysate simultaneously, a gradient was used as the eluent. The flow was $1 \mathrm{~mL} / \mathrm{min}$ with a mixed mobile phase that included solvent A (water with $0.1 \%$ trifluoroaceticacid [TFA]) and solvent B (acetonitrile with $0.1 \%$ TFA). The gradient was as follows: $0-15$ minutes, from $95 \%$ solvent A to $70 \%$ solvent A; $15-25$ minutes, from $70 \%$ solvent A to $100 \%$ solvent $B$. This system was sustained at $100 \%$ solvent B for 5 minutes.

\section{Cellular uptake}

Based on confirmed reports, MCF-7 cells are known to under-secrete MMP-2/9.21,22 MCF-7 cells were selected as low MMP-secreting tumor cell types to investigate the uptake of activable conjugates after extra MMPs trigger cleavage. HT-1080 cells were used as overexpressed MMP-2/916,23 tumor cell types to study the cellular uptake of activable ACPP-DOX after cell-secreted MMPs trigger cleavage. Innate fluorescence of DOX allowed us to use flow cytometry and confocal microscopy to study penetration and localization of free or conjugated DOX.

Collagenase IV or MMP-2 was activated at $37^{\circ} \mathrm{C}$ with the $2.5 \mathrm{mM}$ APMA solution. MCF-7 cells were seeded into six-well plates $\left(2 \times 10^{5}\right.$ cells per well $)$ and allowed to grow for 24 hours. These cells were washed with phosphate-buffered saline (PBS; $0.1 \mathrm{M}, \mathrm{pH}$ 7.4) and preincubated with different concentrations of activated enzyme solutions for 1 hour. Then the supernatants were removed and discarded, and the cells were incubated with $5 \mu \mathrm{M}$ of ACPP-DOX dissolved in $1 \mathrm{~mL}$ of blank culture for 12 hours. After the incubation, the cells were washed three times with PBS (0.1 M, pH 7.4). A total of $1 \mathrm{~mL}$ of $0.25 \%$ trypsin solution was added, and the cells were detached from cell culture by incubation for 3 minutes at $37^{\circ} \mathrm{C}$. The cell suspensions were centrifuged for 3 minutes and resuspended in $0.5 \mathrm{~mL}$ PBS. Single-cell suspensions were prepared by filtration through a 300-mesh filter. Finally, the cells suspended in PBS were subjected to flow cytometry analysis using a FACScan (Becton Dickinson, San Jose, CA). The cells associated with DOX or ACPP-DOX were excited with an argon laser at $488 \mathrm{~nm}$ and fluorescence was detected at $588 \mathrm{~nm}$. Files of 10,000 gated events were collected and analyzed with the FACStation software (Cellquest 3.3) program.

MCF-7 and HT-1080 cells were seeded into six-well plates $\left(2 \times 10^{5}\right.$ cells per well) and allowed to grow for 24 hours. The cells were incubated with free DOX or ACPP-DOX at an equal concentration of $10 \mu \mathrm{M}$ for different periods. After the incubation, the drug solutions were removed and the cells were washed three times with PBS (0.1 M, pH 7.4). Subsequently, the same flow cytometry procedure described above was performed for determining cellular uptake.

Following the culture of MCF-7 and HT-1080 cells for 24 hours on a petri dish, free DOX or ACPP-DOX at a concentration of $5 \mu \mathrm{M}$ was added to each dish and incubated at $37^{\circ} \mathrm{C}$. The medium was removed and discarded, and the cells were washed with PBS (0.1 M, pH 7.4), followed by fixing with $4 \%$ paraformaldehyde for 10 minutes. Nuclear staining was performed by Hoechst 33258 for 15 minutes, and the fluorescent images of cells were analyzed using a laser scanning confocal microscope (TCS SP5; Leica Microsystems $\mathrm{GmbH}$, Wetzlar, Germany).

\section{Antiproliferative activity against MCF-7 and HT-I 080 cells}

The antiproliferative activity of DOX, ACPP-DOX, and ACPP was analyzed by the MTT method. ${ }^{24}$ MCF-7 and HT- 1080 cells were seeded at a density of $2 \times 10^{4}$ cells/well in 96-well plates. After culturing for 24 hours in an incubator $\left(37^{\circ} \mathrm{C}, 5 \% \mathrm{CO}_{2}\right), 20 \mu \mathrm{L}$ of various sample solutions in a concentration range of $1 \mu \mathrm{M}$ to $100 \mu \mathrm{M}$ were added to each well, and the plates were incubated for 24 hours. Next, $20 \mu \mathrm{L}$ of MTT solution $(5.0 \mathrm{mg} / \mathrm{mL})$ was added to each well, and the plates were incubated for another 4 hours. After that, the MTT medium was replaced by $200 \mu \mathrm{L}$ of dimethyl sulfoxide (DMSO) in each well, and the mixture was shaken at room temperature to dissolve the reacted dye. The optical density was measured at $570 \mathrm{~nm}$ using a microplate reader (Bio-Rad, Hercules, CA). Cell viability $=(\mathrm{A}-\mathrm{B}) / \mathrm{A} \times 100 \%$, where $\mathrm{A}$ was the absorbance of the cells incubated with the culture medium and B was the absorbance of the cells incubated with the DOX, ACPP-DOX, or ACPP, respectively. All samples were evaluated in sextuplicate.

\section{Data analysis}

Data were expressed as the mean \pm standard deviation, and statistical analysis was performed using an unpaired 
Student's $t$-test. Data were considered significantly different at $P<0.05$.

\section{Results and discussion Design and synthesis of ACPP-DOX}

For tumor cells to invade and metastasize, the first barrier to overcome is the basement membrane, which is composed of type IV collagen. Type IV collagen is susceptible to degradation by MMP-2 and MMP-9. ${ }^{25}$

$\mathrm{H}_{2}$ N-DGGDGGDGGDGPLGLAG-rrrrrrrrrC-COOH was designed as the ACPP. The ACPP includes three units: the cell-penetrating domain (polyarginine, R9), the cleavable enzyme-specific substrate domain of MMP-2/9 (PLGLAG), and the attenuating peptides domain (DGGDGGDGGDG). Some research indicates that L-polyarginine (R9) was 20-fold more efficient than Tat(49-57) at cellular uptake as determined by Michaelis-Menten kinetic analysis, and the D-polyarginine (R9) exhibited an even greater enhancement of uptake rate ( $>100$-fold) than the R9. ${ }^{26}$ PLGLAG was considered a remarkably sensitive or cleavable sequence by MMP-2 and MMP-9. ${ }^{19,27}$ To prevent CPP distribution toward normal cells, polyanional DGGDGGDGGDG is added to the molecule as an attenuating or shielding motif. The C-terminal cysteine is a linker designed to couple easily with the maleimide group. Thus, the ACPP peptide is temporarily inactivated, and subsequent activation is triggered by overexpressed MMPs in tumor targets where the CPP will be released.

DOX-SMP was synthesized by a reaction between the $3^{\prime}$ amino group of the daunosamine sugar of DOX and an active ester group of SMP. TEA was added to remove the hydrochloride salt and also to maintain basic conditions to favorably generate DOX-SMP. DOX-SMP HPLC analysis: 93.5\%; yield: $64.3 \%$; matrix-assisted laser desorption ionization orthogonal time of flight mass spectrometry (MALDI-TOF-MS) $\left(\mathrm{m} / \mathrm{z},[\mathrm{M}+\mathrm{Na}]^{+}\right): 717.6$ (calculated), 715.95 (observed). MALDI-TOF-MS $\left(\mathrm{m} / z,[\mathrm{M}+\mathrm{K}]^{+}\right): 733.6$ (calculated), 732.03 (observed). ESI-MS $\left(\mathrm{m} / z,[\mathrm{M}+\mathrm{Na}]^{+}\right)$: 717.6 (calculated), 717.1 (observed).

The ${ }^{1} \mathrm{H}-\mathrm{NMR}$ spectrum of DOX-SMP in DMSO-D6 (Bruker, 400M; Zurich, Switzerland) is shown in Figure 1C. O-methylation of the 4-position and 14- $\mathrm{CH}_{2}$ in the DOX-SMP molecule were seen at $3.972 \mathrm{ppm}$ and $4.55 \mathrm{ppm}$ (single peak), respectively. The 6-OH and 11-OH of DOX-SMP were also observed at $14.026 \mathrm{ppm}$ and $13.266 \mathrm{ppm}$, respectively. Chemical shifts between 7.5 and $8.0 \mathrm{ppm}$ were assigned as $1 \mathrm{H}-3 \mathrm{H}$ of the benzene. Furthermore, an obvious single peak at 6.952 ppm was a characteristic shift representing the $1^{\prime}-\mathrm{H}$ and $2^{\prime}-\mathrm{H}$ of the maleimide group. The ${ }^{1} \mathrm{H}-\mathrm{NMR}$ results indicated that the compound was in agreement with the predicted structure of DOX-SMP. In addition, the Fourier transform infrared (FTIR) spectrum of DOX-SMP (Figure 1D) showed that a single peak at $3369.88 \mathrm{~cm}^{-1}$ was attributed to a hydrogen skeletal vibration of a secondary amide group. The appearance of a secondary amide showed that DOX and SMP were coupled by an amide linkage. All of the above characteristics demonstrated that DOX-SMP was synthesized successfully.

The C-terminal amino acid of the ACPP peptide is a cysteine, which provides a chemically reactive thiol group for drug conjugation. DOX-SMP can be covalently attached to the cysteine sulfur of ACPP with maleimide by way of a Michael addition (nucleophilic addition) to produce ACPP-DOX. HPLC analysis: 95.3\%; yield: 78.8\%; MALDI-TOF-MS $\left(\mathrm{m} / \mathrm{z},[\mathrm{M}-\mathrm{H}]^{-}\right): 3588.8$ (calculated), 3587.26 (observed). Thus, according to the well-designed synthetic route and proven by the MALDI-TOF-MS result, a derivative of DOX linked with ACPP (ACPP-DOX) was obtained successfully.

\section{Enzymatic-triggered cleavage of ACPP-DOX by MMPs}

ACPP-DOX incorporates a MMP-2/9-sensitive substrate; subsequently, the triggered cleavage study was performed through an extra enzymatic digestion experiment on different [MMPs]/[ACPP-DOX] ratios for 4 hours. As MMP concentration decreased, ACPP-DOX cleavage increased proportionally (Figure 2). The half-lives of ACPP-DOX cleavage were approximately 0.6 hour, 0.9 hour, 1.1 hours, and 2.5 hours with a series of (MMPs)/(ACPP-DOX) ratios of $0.463: 1,0.288: 1,0.173: 1$, and $0.114: 1$, respectively. In contrast, almost no cleavage was observed for ACPP-DOX

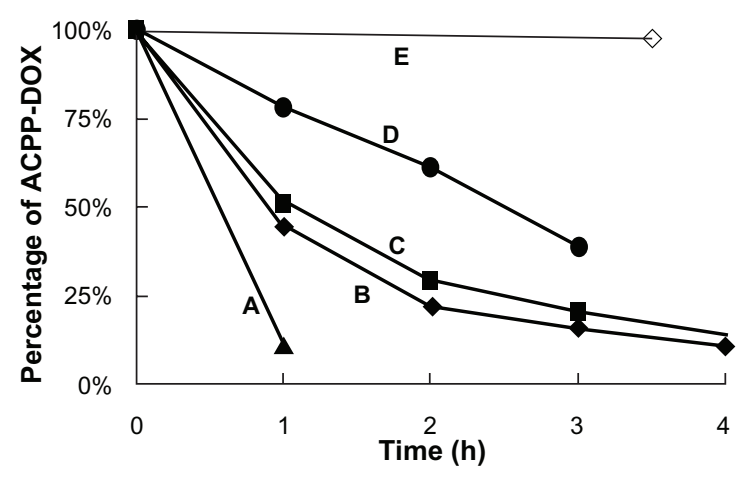

Figure 2 Enzymatic-triggered cleavage of ACPP-DOX conjugate by MMPs in different ratios.

Note: $(\mathrm{MMPs}) /($ ACPP-DOX) $(w / w)$ of $($ (A) $0.463: 1 \quad$ (B) $0.288: 1$ (C) $0.173: 1$ (D) $0.114: 1$, and (E) absence of MMP treatment.

Abbreviations: ACPP-DOX, activable cell-penetrating peptide-doxorubicin; MMP, matrix metalloproteinase. 
in the absence of MMPs after 3.5 hours of incubation. The experiment results proved that MMP-2/9 can cleave the ACPP and the scissor rate of ACPP-DOX was related to the concentration of MMP-2/9. MMP concentration was obviously related to the degree of cleavage; more cleavage was also found in MMP-activated imaging nanoparticles with the increase of extra MMPs. ${ }^{28}$ In our previous experiments, the hydrolysis fragments of ACPP have been detected by MALDI-TOF-MS and it was proved that the ACPP could be cleaved by MMP-2/9 at target site. ${ }^{29}$

\section{Enhancing cellular uptake after enzymatic- triggered cleavage of ACPP-DOX}

When ACPP-DOX is scissored and activated to CPP-DOX by MMP-2/9, cellular uptake of CPP-DOX is expected to be enhanced due to the effect of CPP penetration. MCF-7 cells were used as a model system of under-expressed MMP-2/9 to study the effect of extra MMP-triggered cleavage of ACPP-DOX on cellular uptake. As shown in Figure 3A, MCF-7 cell-associated fluorescence was significantly higher (5.1- or 5.6-fold) in either group after ACPP-DOX activation by MMP-2/9 than that of the group with absence of collagenase $(P<0.001)$. It was obvious that the increase in MMP-2/9 concentration from 20 to $200 \mu \mathrm{g} / \mathrm{mL}$ resulted in stronger uptake of DOX $(P<0.05)$. MMP-2 pretreatment was performed in MCF-7 cells (Figure 3B). ACPP-DOX showed an approximately 3.4-fold higher uptake when the concentration of MMP-2 was $100 \mathrm{ng} / \mathrm{mL}$ compared with the group without MMP-2 $(P<0.001)$. In contrast, $20 \mathrm{ng} / \mathrm{mL}$ of MMP-2 treatment had a lower uptake of ACPP-DOX compared to the group treated with $100 \mathrm{ng} / \mathrm{mL}$ of MMP-2 $(P<0.001)$.

HT-1080 cells were used as a model system of overexpressed MMP-2/9 to study the effect of triggered cleavage of ACPP-DOX on cellular uptake. As shown in Figure 3C, fluorescence intensity of free DOX in both MCF-7 and HT-1080 cells was similar after 8 hours of incubation. Cellular uptake properties of free DOX were in accordance with the reported results and this might be responsible for transportation into cells by the passive diffusion mechanism. ${ }^{30,31}$ Compared with free DOX, ACPP-DOX displayed lower uptake into both cells. However, it was noteworthy that the uptake intensity of ACPP-DOX was significantly higher in HT-1080 cells than in MCF-7 cells after 24 hours of incubation. After 48 hours of incubation, the uptake intensity of ACPP-DOX in HT-1080 cells was 3.3-fold higher than that in MCF-7 cells. This implied that ACPPDOX was more sensitive to HT-1080 than to MCF-7 cells, and the sensitivity corresponded with the HT-1080 cell over-secreted MMPs.

The intracellular trafficking of DOX and ACPP-DOX was studied in HT-1080 and MCF-7 cells using confocal laser scanning microscopy. Different incubation times were employed from 2 to 8 hours (Figure 3D). The uptake was weak in both HT-1080 and MCF-7 cells treated with ACPP-DOX for 2 hours. The fluorescence emerged at 4 hours and gradually increased with longer incubation. The fluorescence intensity was significantly stronger in HT-1080 cells than in MCF-7 cells over time, which meant that more DOX entered HT-1080 cells. For DOX, its fluorescence was clearly observed in HT-1080 and MCF-7 cells after incubation for only 2 hours, a longer incubation time ( 4 or 8 hours) resulted in stronger fluorescence.

It was noteworthy that the degree of uptake enhancement was obviously related to the extra MMP-2/9 trigger on MCF-7 cells and the endogenous MMP trigger on HT-1080 cells. In addition, the higher uptake of nanostructured lipid carriers incorporating ACPP on HT-1080 cells was inhibited in the presence of GM6001 (Enzo Life Sciences, Inc, Farmingdale, NY), a known MMP inhibitor (data not shown). These phenomena were in accordance with extra enzymatic cleavage analysis as determined by HPLC (Figure 2) and suggested that the cleavage was specifically by MMPs. ${ }^{16,32-34}$ Enhanced cellular uptake is important after triggered activation especially for some poor membranepenetrating candidates.

\section{Antiproliferative activity}

To examine the effect of ACPP-DOX, DOX, and ACPP on cell growth and viability, the potential antiproliferative activities were measured by the MTT method on HT-1080 and MCF-7 cells. For parallel comparison, cells were incubated with ACPP-DOX, DOX, and ACPP at a series of equivalent concentrations from 1 to $100 \mu \mathrm{M}$. As shown in Figure 4, the ACPP did not show any obvious antiproliferative activities in HT-1080 and MCF-7 cells after 24 hours of incubation, which revealed that the ACPP was relatively safe. With increased concentration of DOX, the antiproliferative activities of free DOX increased significantly on both MCF-7 and HT-1080 cells, but the ACPP-DOX conjugate displayed stronger antiproliferative activities on HT-1080 than on MCF-7 cells. Namely, the ACPP-DOX conjugate was more effective against HT-1080 than MCF-7 cells correlating to the level of MMPs, despite the fact that DOX had almost equal cytotoxicity both on HT-1080 and MCF-7 cells with no correlation to the proteases. 

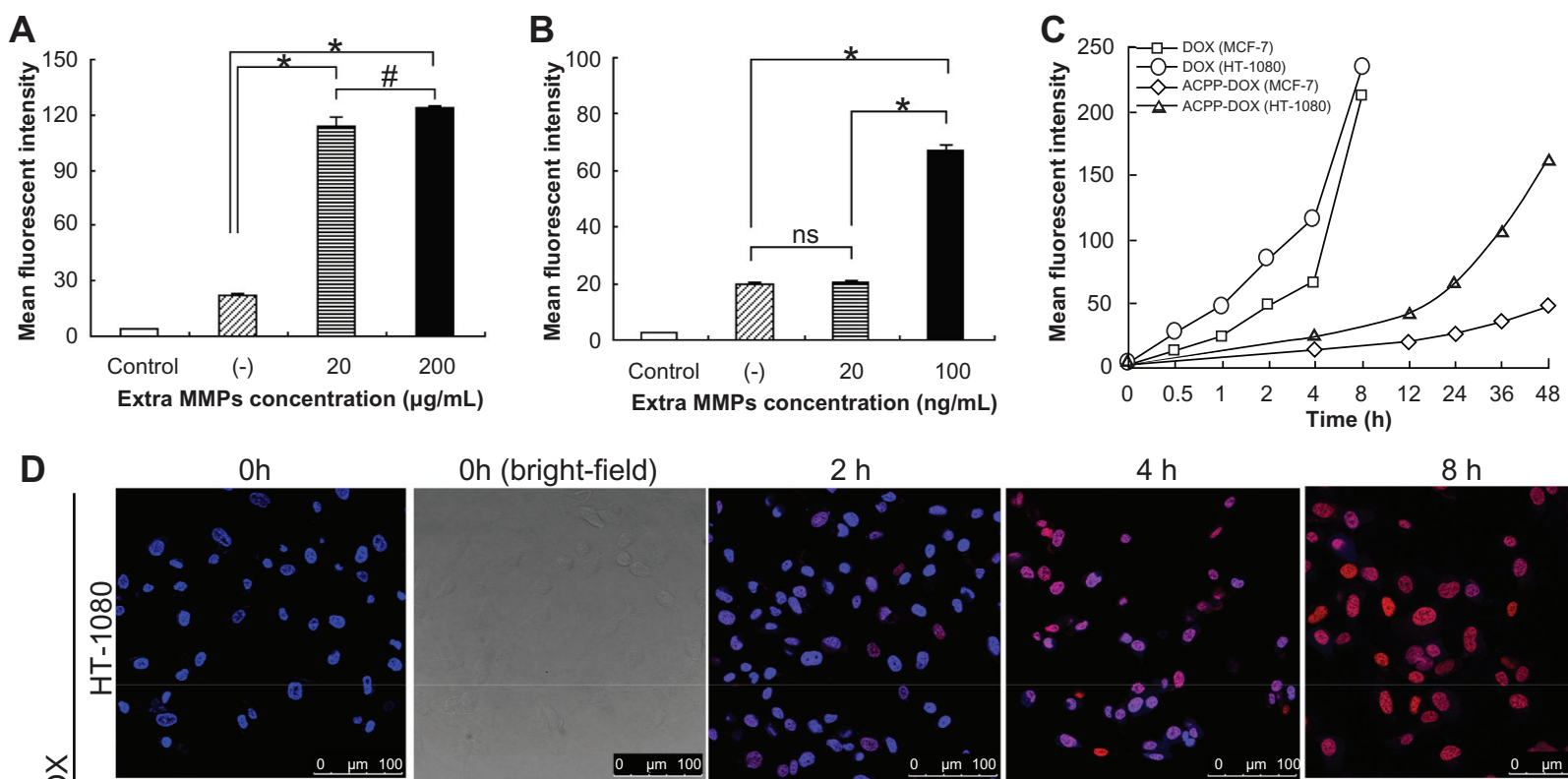

Oh (bright-field)

$2 \mathrm{~h}$

$4 \mathrm{~h}$

$8 \mathrm{~h}$
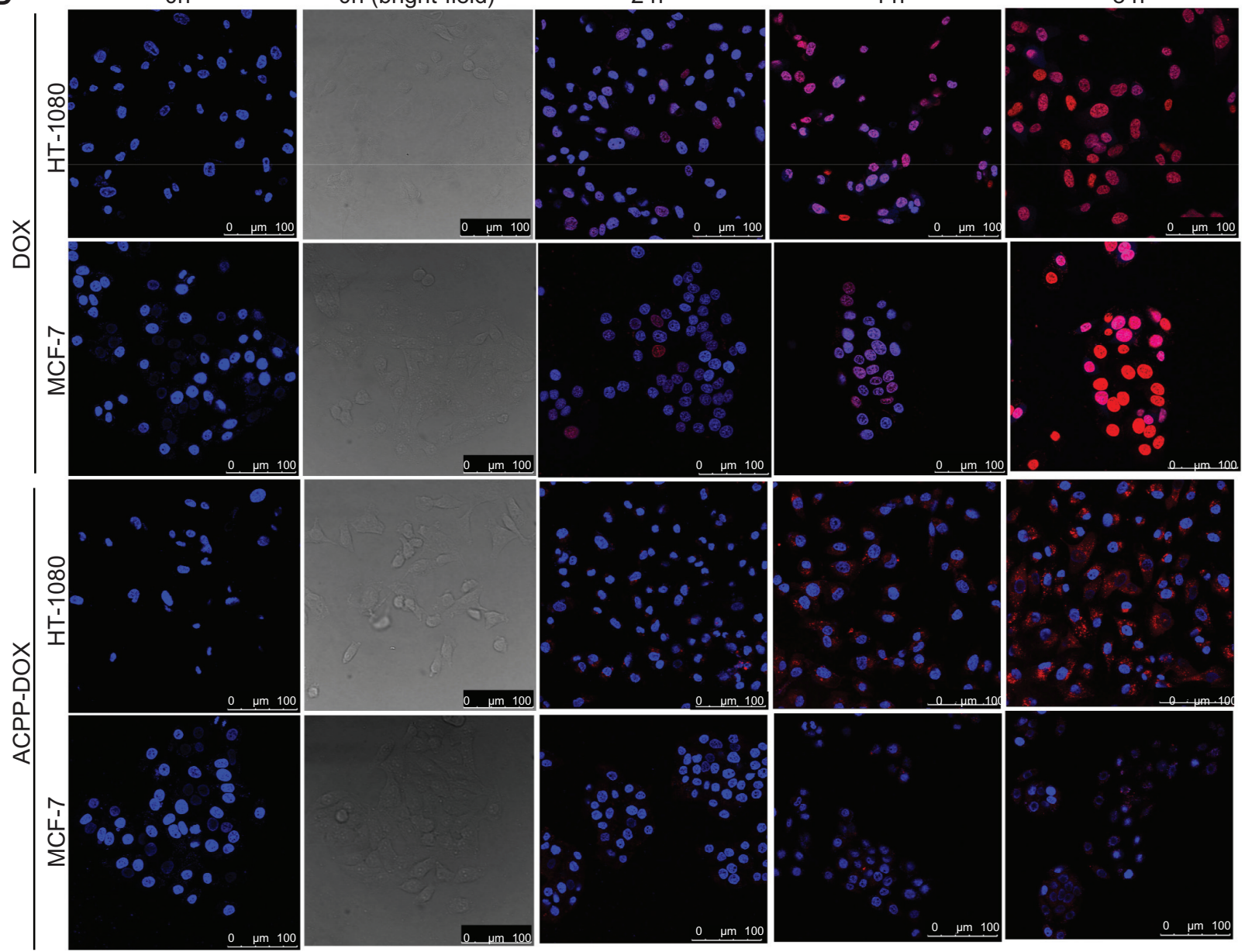

Figure 3 (A) The uptake intensity of MCF-7 cells after treatment with ACPP-DOX conjugate in the absence $(-)$ or presence of extra collagenase IV at concentrations of $20 \mu \mathrm{g} / \mathrm{mL}$ and $100 \mu \mathrm{g} / \mathrm{mL}$. (B) The uptake intensity of MCF-7 cells after treatment with ACPP-DOX conjugate in the absence (-) or presence of extra MMP-2 at concentrations of $20 \mathrm{ng} / \mathrm{mL}$ and $200 \mathrm{ng} / \mathrm{mL}$. (C) Cellular uptake intensity-time curve of DOX and ACPP-DOX in MCF-7 and HT-1080 cells at different incubation times. (D) Confocal microscopy images of ACPP-DOX or DOX incubated with HT- 1080 and MCF-7 cells at $37^{\circ} \mathrm{C}$ for 0 hour- 8 hours.

Notes: Red represents fluorescence of DOX. Blue represents fluorescence of Hoechst 33258 for nuclei staining. Scale bars: $100 \mu \mathrm{m}$. $* P<0.00$ I; $\#<0.05 ;$ ns $=$ no significance.

Abbreviations: DOX, doxorubicin; ACPP-DOX, activable cell-penetrating peptide-doxorubicin; MMP-2, matrix metalloproteinase-2.

The increased cytotoxicity of ACPP-DOX conjugate toward HT-1080 cells was probably due to released CPP-DOX and enhanced cell uptake by the activation of overexpressed MMP-2/9 in this cell type. Our previous cell-uptake studies of CPP-cargos have demonstrated that polyarginine peptides as well as CPP-avidin can easily enter cells and tissues. ${ }^{12}$ Abundant research proves that CPPs could transport cargos into cells. ${ }^{1-5,35-37}$ Numerous small molecule chemotherapeutics, such as taxol, ${ }^{38}$ methotrexate, ${ }^{10}$ and $\mathrm{DOX}^{8,11}$ have shown improved activity when conjugated to 


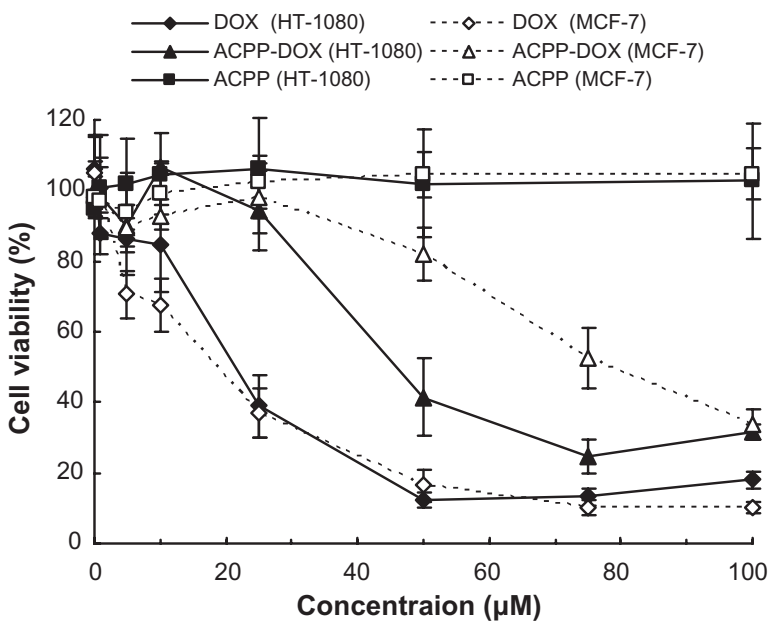

Figure 4 Antiproliferative activity of DOX, ACPP-DOX, and ACPP against MCF-7 and HT-I080 cells.

Notes: Antiproliferative activity was determined using the MTT method after incubation for 24 hours in vitro. Data are represented as the mean \pm standard deviation $(n=6)$

Abbreviations: DOX, doxorubicin; ACPP-DOX, activable cell-penetrating peptidedoxorubicin; MTT, 3-(4,5-dimethylthiazol-2-yl)-2,5-diphenyltetrazolium bromide.

a CPP. Remarkably, even though a CPP-drug conjugate may show less activity in a purified biochemical system, highly efficient cellular uptake can overcome this shortfall, because CPP-drug conjugates can effectively increase the intracellular concentrations of drug molecules and this can counteract decreases in drug activity that result from conjugation. ${ }^{10,14}$ These results indicate that ACPP-drug conjugate delivery strategy provides potential for tumor-targeted delivery of antitumor therapeutics by triggered activation.

\section{Conclusion}

The ACPP approach could potentially address the main issue that CPPs lack cell specificity by means of the selective trigger of proteases at the tumor targets. To our knowledge, reported ACPPs were mostly designed as imaging agents. We synthesized ACPP-DOX conjugate in good purity and yields. The proteolytic activation of the ACPP-DOX conjugate occurs in an enzyme concentration-dependent manner. The enhanced cellular uptake and antiproliferative activity of ACPP-DOX conjugate was observed after MMP-senstive activation and revealed that ACPP-DOX conjugate has targeting ability for tumor cells rich in MMP-2/9. The ACPPdrug conjugate delivery system triggered by protease is a promising approach for the treatment of some tumors with overexpression of MMP-2 or MMP-9.

\section{Acknowledgments}

This work was supported by the National Natural Science Foundation of China (30772665 and 30970785), National
Basic Research Program of China (2007CB935801 and 2009CB930300), and the Doctoral Foundation of the Ministry of Education of China (20100001110056).

\section{Disclosure}

The authors report no conflicts of interest in this work.

\section{References}

1. Fawell S, Seery J, Daikh Y, et al. Tat-mediated delivery of heterologous proteins into cells. Proc Natl Acad Sci U S A. 1994;91(2):664-668.

2. Chiu YL, Ali A, Chu CY, Cao H, Rana TM. Visualizing a correlation between siRNA localization, cellular uptake, and RNAi in living cells. Chem Biol. 2004;11(8):1165-1175.

3. Rothbard JB, Garlington S, Lin Q, et al. Conjugation of arginine oligomers to cyclosporin A facilitates topical delivery and inhibition of inflammation. Nat Med. 2000;6(11):1253-1257.

4. Santra S, Yang H, Stanley JT, et al. Rapid and effective labeling of brain tissue using TAT-conjugated CdS:Mn/ZnS quantum dots. Chem Commun (Camb). 2005;7(25):3144-3146.

5. Lewin M, Carlesso N, Tung CH, et al. Tat peptide-derivatized magnetic nanoparticles allow in vivo tracking and recovery of progenitor cells. Nat Biotechnol. 2000;18(4):410-414.

6. Mazel M, Clair P, Rousselle C, et al. Doxorubicin-peptide conjugates overcome multidrug resistance. Anticancer Drugs. 2001; 12(2):107-116

7. Liang JF, Yang VC. Synthesis of doxorubicin-peptide conjugate with multidrug resistant tumor cell killing activity. Bioorg Med Chem Lett. 2005;15(22):5071-5075.

8. Aroui S, Brahim S, De Waard M, Bréard J, Kenani A. Efficient induction of apoptosis by doxorubicin coupled to cell-penetrating peptides compared to unconjugated doxorubicin in the human breast cancer cell line MDA-MB 231. Cancer Lett. 2009;285(1):28-38.

9. Aroui S, Brahim S, Hamelin J, De Waard M, Bréard J, Kenani A. Conjugation of doxorubicin to cell penetrating peptides sensitizes human breast MDA-MB 231 cancer cells to endogenous TRAILinduced apoptosis. Apoptosis. 2009;14(11):1352-1365.

10. Lindgren M, Rosenthal-Aizman K, Saar K, et al. Overcoming methotrexate resistance in breast cancer tumour cells by the use of a new cell-penetrating peptide. Biochem Pharmacol. 2006;71(4):416-425.

11. Aroui S, Brahim S, Waard MD, Kenani A. Cytotoxicity, intracellular distribution and uptake of doxorubicin and doxorubicin coupled to cell-penetrating peptides in different cell lines: a comparative study. Biochem Biophys Res Commun. 2010;391(1):419-425.

12. Ma DX, Shi NQ, Qi XR. Distinct transduction modes of arginine-rich cell-penetrating peptides for cargo delivery into tumor cells. Int $J$ Pharm. 2011;419(1-2):200-208

13. Vivès E, Schmidt J, Pèlegrin A. Cell-penetrating and cell-targeting peptides in drug delivery. Biochim Biophys Acta. 2008;1786(2): 126-138.

14. Fonseca SB, Pereira MP, Kelley SO. Recent advances in the use of cell-penetrating peptides for medical and biological applications. $A d v$ Drug Deliv Rev. 2009;61(11):953-964.

15. Law B, Tung CH. Proteolysis: a biological process adapted in drug delivery, therapy, and imaging. Bioconjug Chem. 2009;20(9):1683-1695.

16. Jiang T, Olson ES, Nguyen QT, Roy M, Jennings PA, Tsien RY. Tumor imaging by means of proteolytic activation of cell-penetrating peptides. Proc Natl Acad Sci U SA. 2004;101(51):17867-17872.

17. Aguilera TA, Olson ES, Timmers MM, Jiang T, Tsien RY. Systemic in vivo distribution of activatable cell penetrating peptides is superior to that of cell penetrating peptides. Integr Biol (Camb). 2009;1(5-6): 371-381.

18. Olson ES, Aguilera TA, Jiang T, et al. In vivo characterization of activatable cell penetrating peptides for targeting protease activity in cancer. Integr Biol (Camb). 2009;1(5-6):382-393. 
19. Olson ES, Jiang T, Aguilera TA, et al. Activatable cell penetrating peptides linked to nanoparticles as dual probes for in vivo fluorescence and MR imaging of proteases. Proc Natl Acad Sci U S A. 2010;107(9): 4311-4316.

20. Nguyen QT, Olson ES, Aguilera TA, et al. Surgery with molecular fluorescence imaging using activatable cell-penetrating peptides decreases residual cancer and improves survival. Proc Natl Acad Sci US A. 2010;107(9):4317-4322.

21. Giambernardi TA, Grant GM, Taylor GP, et al. Overview of matrix metalloproteinase expression in cultured human cells. Matrix Biol. 1998;16(8):483-496

22. Das S, Banerji A, Frei E, Chatterjee A. Rapid expression and activation of MMP-2 and MMP-9 upon exposure of human breast cancer cells (MCF-7) to fibronectin in serum free medium. Life Sci. 2008;82(9-10): 467-476.

23. Albright CF, Graciani N, Han W, et al. Matrix metalloproteinase-activated doxorubicin prodrugs inhibit HT1080 xenograft growth better than doxorubicin with less toxicity. Mol Cancer Ther. 2005;4(5):751-760.

24. Tian Q, Zhang CN, Wang XH, et al. Glycyrrhetinic acid-modified chitosan/poly(ethylene glycol) nanoparticles for liver-targeted delivery. Biomaterials. 2010;31(17):4748-4756.

25. Murphy G, Nagase H. Progress in matrix metalloproteinase research. Mol Aspects Med. 2008;29(5):290-308.

26. Wender PA, Mitchell DJ, Pattabiraman K, Pelkey ET, Steinman L, Rothbard JB. The design, synthesis, and evaluation of molecules that enable or enhance cellular uptake: peptoid molecular transporters. Proc Natl Acad Sci U S A. 2000;97(24):13003-13008.

27. Kline T, Torgov MY, Mendelsohn BA, Cerveny CG, Senter PD. Novel antitumor prodrugs designed for activation by matrix metalloproteinases-2 and -9. Mol Pharm. 2004;1(1):9-22.

28. Smith R, Sewell SL, Giorgio TD. Proximity-activated nanoparticles: in vitro performance of specific structural modification by enzymatic cleavage. Int J Nanomedicine. 2008;3(1):95-103.
29. Wang PC, Qi XR. The design and enzymatic hydrolysis of activatable cell-penetrating peptide. Yao Xиe Xие Bao. 2010;45(8):1048-1051.

30. Sun Y, Yan X, Yuan T, et al. Disassemblable micelles based on reduction-degradable amphiphilic graft copolymers for intracellular delivery of doxorubicin. Biomaterials. 2010;31(27):7124-7131.

31. Li YL, Zhu L, Liu Z, et al. Reversibly stabilized multifunctional dextran nanoparticles efficiently deliver doxorubicin into the nuclei of cancer cells. Angew Chem Int Ed Engl. 2009;48(52):9914-9918.

32. Terada T, Iwai M, Kawakami S, Yamashita F, Hashida M. Novel PEGmatrix metalloproteinase-2 cleavable peptide-lipid containing galactosylated liposomes for hepatocellular carcinoma-selective targeting. J Control Release. 2006;111(3):333-342.

33. Neumann U, Kubota H, Frei K, Ganu V, Leppert D. Characterization of Mca-Lys-Pro-Leu-Gly-Leu-Dpa-Ala-Arg- $\mathrm{NH}_{2}$, a fluorogenic substrate with increased specificity constants for collagenases and tumor necrosis factor converting enzyme. Anal Biochem. 2004;328(2): $166-173$.

34. Liu S, Netzel-Arnett S, Birkedal-Hansen H, Leppla SH. Tumor cellselective cytotoxicity of matrix metalloproteinase-activated anthrax toxin. Cancer Res. 2000;60(21):6061-6067.

35. Bullok KE, Dyszlewski M, Prior JL, Pica CM, Sharma V, Piwnica-Worms D. Characterization of novel histidine-tagged Tat-peptide complexes duallabeled with (99 m)Tc-tricarbonyl and fluorescein for scintigraphy and fluorescence microscopy. Bioconjug Chem. 2002;13(6):1226-1237.

36. Robbins PB, Oliver SF, Sheu SM, Goodnough JB, Wender P, Khavari PA. Peptide delivery to tissues via reversibly linked protein transduction sequences. Biotechniques. 2002;33(1):190-192, 194.

37. Torchilin VP, Levchenko TS. TAT-liposomes: a novel intracellular drug carrier. Curr Protein Pept Sci. 2003;4(2):133-140.

38. Dubikovskaya EA, Thorne SH, Pillow TH, Contag CH, Wender PA. Overcoming multidrug resistance of small-molecule therapeutics through conjugation with releasable octaarginine transporters. Proc Natl Acad Sci U S A. 2008;105(34):12128-12133.
International Journal of Nanomedicine

\section{Publish your work in this journal}

The International Journal of Nanomedicine is an international, peerreviewed journal focusing on the application of nanotechnology in diagnostics, therapeutics, and drug delivery systems throughou the biomedical field. This journal is indexed on PubMed Central,

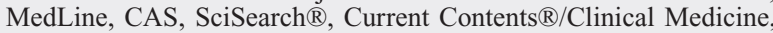

\section{Dovepress}

Journal Citation Reports/Science Edition, EMBase, Scopus and the Elsevier Bibliographic databases. The manuscript management system is completely online and includes a very quick and fair peer-review system, which is all easy to use. Visit http://www.dovepress.com/ testimonials.php to read real quotes from published authors. 